Dicenda. Estudios de lengua y literatura españolas

ISSN-e: $1988-2556$

http://dx.doi.org/10.5209/DICE.62142

\title{
Los prefijos adjetivales: un grupo heterogéneo
}

\author{
Antonio Fábregas ${ }^{1}$
}

Recibido: 29 de marzo de 2016 / Aceptado: 4 de julio de 2017

Resumen. Este artículo discute una de las clases reconocidas de prefijos en español, los prefijos adjetivales. Esta clase muestra propiedades que no encajan con el resto de los prefijos, tanto en su fonología como en su semántica. Se argumenta que la clase es heterogénea y deben distinguirse en ella al menos cuatro subtipos de elementos. Sus propiedades excepcionales, junto a su ordenamiento lineal y su selección categorial, se siguen naturalmente si se consideran especificadores de proyecciones funcionales que forman parte de la secuencia extendida de una base nominal.

Palabras clave: prefijación; modificación; composición; prefijos adjetivales.

\section{[en] Adjectival prefixes: a heterogeneous group}

Abstract. This article discusses one of the identified classes of prefixes in Spanish, adjectival prefixes. This class displays a behaviour that is exceptional among prefixes, including their phonology and semantics. We argue that the class is actually heterogeneous, and that at least four subclasses must be differentiated inside them. Their exceptional properties, as well as their linear ordering and category selection, follow if they are treated as specifiers of functional projections that belong to the extended sequence of a nominal base.

Keywords: prefixation; modification; compounding; adjectival prefixes.

Sumario: 1. Problemas de la prefijación; 2. La prefijación adjetival; 2.1. Propiedades fonológicas; 2.2. Propiedades semánticas; 2.3. ¿Por qué no son compuestos?; 3. Subclases de prefijos adjetivales; 3.1. Propiedades compartidas; 3.1.1. Orden; 3.1.2. Categoría gramatical de la base; 3.1.3. Generalizaciones semánticas; 3.2. Propiedades diferenciadoras; 3.2.1. Propiedad de la base vs. propiedad de un pseudo-argumento de la base; 3.2.2. Uso como adjetivo independiente; 3.2.3. Modificación de adjetivos calificativos y nombres propios; 3.2.4. Orden relativo entre los prefijos; 4. Análisis: generalizaciones y propiedades; 4.1. Micro-: su naturaleza estructural; 4.2. Neo-: su naturaleza estructural; 4.3. Pseudo-: su naturaleza estructural; 5. Conclusiones: consecuencias para la división entre composición y prefijación.

Cómo citar: Fábregas, A. (2018). Los prefijos adjetivales: un grupo heterogéneo, en Dicenda. Estudios de Lengua y Literatura Españolas, 36, 167-189.

Departamento de lengua y cultura, Universidad de Tromsø

antonio.fabregas@uit.no 


\section{Problemas de la prefijación}

Más allá del nivel descriptivo, son muchas las preguntas que surgen del examen de la prefijación en español (cf. Almela, 1999; Montero Curiel, 1999; Varela \& Martín García, 1999; Stehlík 2011), pero tal vez las dos más tradicionales son las siguientes:

¿Cuáles son los límites entre la prefijación y la composición?

¿Qué determina que un morfema sea prefijo y no sufijo?

Acerca de la primera cuestión, muchas veces se ha abordado desde una perspectiva funcional que da por hecho una noción no ambigua de palabra. Así, una buena parte de la bibliografía ha presentado la controversia desde la perspectiva de si una palabra formada por lo que parece una preposición y, por ejemplo, un sustantivo (1) ha de considerarse compuesta, toda vez que la preposición sería una forma que puede citarse independientemente.

\section{(1) ante-ojo}

No es este el aspecto de la polémica que nos interesa, en la medida en que una definición de compuesto que se apoye en la supuesta existencia de dos o más formas libres, llámense palabras o no, ha mostrado ser insuficiente (cf. Scalise et al. 2005; Fábregas \& Scalise 2010, o Lieber \& Stekauer 2009, donde se proporcionan conjuntos de propiedades independientes de si el compuesto contiene o no formas autónomas, sea lo que sea que la autonomía quiera decir en el caso de las preposiciones, que de todos modos requieren un constituyente nominal para poder funcionar como unidades en sintaxis).

El problema existe, pero es mucho más profundo de lo que la supuesta dificultad de categorizar (1) sugiere. La composición establece relaciones semánticas muy determinadas entre los miembros del compuesto, sean raíces, temas o unidades de nivel superior: coordinación (verdinegro), subordinación (telaraña) o atribución (pelirrojo). La prefijación, en principio, rechaza estas interpretaciones, y generalmente viene asociada a significados en los que el prefijo parece operar sobre alguna noción de la base, especificando algunos de sus valores: la localización temporal (pre-análisis) o espacial (intra-venoso), el grado (ultracatólico), la cantidad (bi-plaza) o simplemente la negación (in-útil), reversión (des-hacer) o privación (a-legal) de lo expresado en la base, entre otros muchos valores (cf. RAE \& ASALE, 2009: §10).

¿Por qué habría de ser esto así? ¿Es la división entre compuestos y prefijos real o solo aparente? ¿Es una coincidencia que estos valores se hayan repartido así, o hay algún motivo profundo que relacione la naturaleza de la prefijación y la composición con sus valores respectivos? El problema viene agravado por el hecho de que ciertos casos clasificados como ‘prefijación' sí parecen mostrar una relación atributiva entre los dos morfemas. Este es el caso de algunos de los llamados 'prefijos adjetivales', a los que dedicaremos este trabajo.

La segunda pregunta enfrenta la prefijación con la sufijación. Es cierto que nunca ha habido polémicas de clasificación entre prefijos y sufijos, esencialmente porque se han distinguido mediante el criterio superficial de la posición relativa con respecto a una raíz: si están a la derecha, son sufijos; si están a la izquierda, son prefijos. No 
obstante, en español se ha observado que prefijos y sufijos desempeñan prototípicamente papeles distintos: los sufijos son capaces de alterar la categoría gramatical de la base (2a), expresar sus accidentes morfológicos (2b) y definir propiedades centrales en morfología como la clase morfológica del sustantivo o la conjugación del verbo (2c). Los prefijos españoles no son capaces de hacer esto, si bien existen lenguas en las que sí se documentan al menos cambios de categoría y expresión de accidentes morfológicos mediante prefijación.
(2) a. corr-e-dor
b. cant-á-ba-mos
c. clas-ific-a(r) [1 a conjugación]; gan-a-nci-a [femenino]

No obstante, esta caracterización general da lugar a una pregunta: si esto es cierto empíricamente, ¿el hecho de que algo sea prefijo se sigue de sus funciones en la formación de palabras o tiene que ser estipulado para cada morfema si se materializa a la derecha o a la izquierda de la raíz? ¿Puede decirse que la posición de un morfema depende de sus valores gramaticales, como sugiere, por ejemplo DiSciullo (2005) cuando identifica los prefijos con operadores? El dilema se agrava cuando reparamos en que hay sufijos que, al igual que prefijos cuasi sinónimos a ellos, pueden expresar grado o expresan nociones semánticas que no vienen acompañadas de cambio categorial, no corresponden a accidentes gramaticales con incidencia sobre la distribución formal de la palabra y no alteran las propiedades morfológicas de la base:
(3) a. super-guap-o; guap-ísim-o
b. os-o; os-ezn-o

El objetivo de este trabajo es esencialmente programático. Su marco empírico se encuentra en el examen de la clase de prefijos llamados 'prefijos adjetivales'; examinaremos sus propiedades, que resultan sumamente problemáticas desde la perspectiva de la clasificación entre composición y prefijación (§2). Seguidamente, argumentaremos que estos elementos deben ser divididos en subgrupos cuyas propiedades son distintas ( 33$)$. A continuación, con el objetivo de entender qué nos dicen sobre las dos preguntas anteriores sus propiedades, tomaremos un representante de cada clase y determinaremos su posición morfosintáctica en la sección $\S 4$; la sección $\S 5$ extrae las consecuencias teóricas fundamentales que estos elementos aportan al debate sobre la definición de composición frente a prefijación, y la posibilidad de derivar la posición de un morfema a partir de sus propiedades internas.

\section{La prefijación adjetival}

En RAE \& ASALE (2009: §10.12) se discute una clase de morfemas, clasificados como prefijos por motivos que se expondrán a continuación, a los que se les da la etiqueta de 'adjetivales' porque "los significados que corresponden [a ellos] son mucho más específicos” que aquellos que corresponden a los prefijos clásicos (como pre-, contra- o $s u b-)$ y son nociones que normalmente se expresan con adjetivos en 
español. Esta definición es un buen punto de partida para analizar esta clase, en la que entran los prefijos de (4).

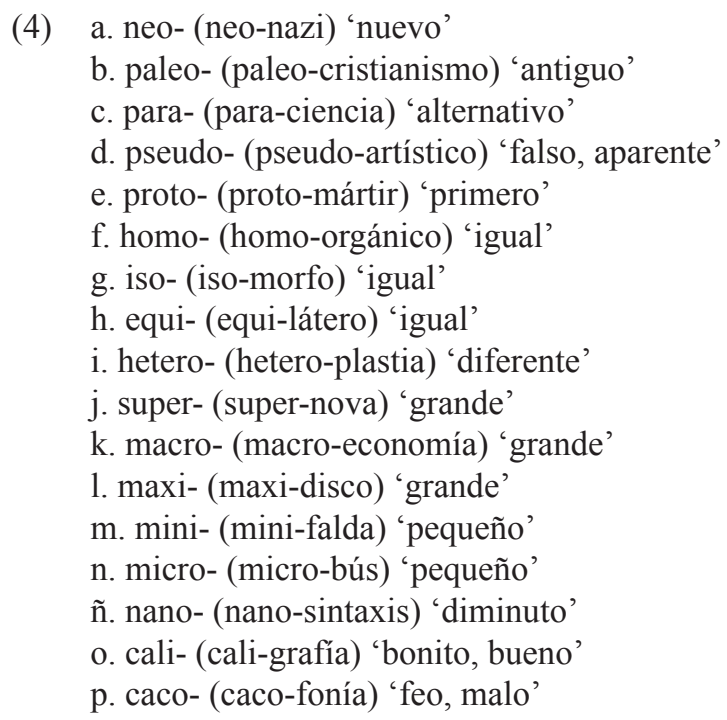

No obstante, obsérvese que los criterios que emplea esta definición, en principio, son menos precisos que los que definen a otras clases de prefijos, como los negativos o los de cantidad. El papel que desempeñan se define como similar al de algunos adjetivos, pero los adjetivos, como sabemos, forman una clase relativamente heterogénea. Sería conveniente, por tanto, tener criterios más formales que permitieran identificar a los miembros de la clase, y ver qué puntos de contacto tienen con otras clases de prefijación. Este es el objetivo de este trabajo, el de examinar las propiedades formales de estos prefijos y así determinar qué criterios permiten agruparlos. De hecho, veremos en este trabajo que hay al menos un prefijo clasificado en otra clase, semi-, que despliega propiedades semejantes a las de los miembros de esta clase.

RAE \& ASALE observan (2009: $§ 10.1 d$ ) que hay una polémica acerca de si las palabras formadas por estos elementos se deben considerar prefijadas o compuestas; el debate se presenta allí como un resultado de si se atiende más a la etimología de los formantes, que en latín o griego solían corresponder a adjetivos independientes, o a la estructura de la palabra. La primera perspectiva favorecería un análisis como compuestos, mientras que la segunda iría en la línea de un análisis de prefijación. No obstante, en los párrafos que siguen vamos a argumentar que, incluso si se adopta una perspectiva puramente sincrónica y se evita toda referencia al origen histórico de las formas, hay motivos estructurales que resultan problemáticos para la adscripción de estas formaciones a una de las dos categorías.

\subsection{Propiedades fonológicas}

Comencemos con una propiedad fonológica. Si pensamos en el fenómeno del acento rítmico en español, es conocido que las palabras polisílabas definen, a partir del acento primario de la palabra, acentos secundarios en sílabas alternas: 

(5) a. Deu.tè.ro.nó.mio
b. es.tèr.no.clèi.do.màs.toi.dé.o
c. plus.cuàm.per.féc.to

Este acento rítmico sigue las reglas que se esperan si se construye definiendo pies métricos binarios dentro de los cuales solo se acentúa una de las dos sílabas, regularmente la primera:

\section{(6) (es)(tèr.no)(clèi.do)(màs.toi)(dé) $<0>$}

Los compuestos, por contra, conservan otra clase de acento no primario: mientras que el miembro a la derecha lleva el acento primario, el segundo miembro conserva, como acento secundario, acento en la sílaba que le abría correspondido de aparecer como una forma libre. Esto se comprueba, por ejemplo, en (7), donde la vocal de la base cont- diptonga en el interior del compuesto, como si llevara el acento primario que se asocia a la misma diptongación en la conjugación verbal:
(7) a. cuèn.ta.cuén.tos
b. cuén.ta vs. * cónt.a
c. con.tá.mos vs. *cuen.tá.mos

Este fenómeno es distinto del acento rítmico de las palabras sin estructura compositiva. El acento rítmico asigna acento a las sílabas de forma alterna -siempre entre dos acentos hay una sílaba átona (8)-, mientras que este acento secundario puede darse en una sílaba adyacente a la que lleva el acento primario (9) o dejar dos sílabas sin acento entre ella y el primario (10).
(8) *es.tèr.no.clei.dò.mas.tòi.dé.o
(9) a.zùl.grá.na (*à.zul.grá.na)
(10) ciu.dàd.dor.mi.tó.rio (*ciù.dad.dòr.mi.tó.rio)

En los prefijos prototípicos, se aplica la regla del acento rítmico:

$$
\begin{aligned}
& \text { (11) a. in.ú.til (*ìn.ú.til) } \\
& \text { b. sùb.lu.nár (*sub.lu.nár) } \\
& \text { c. mùl.ti.mì.llo.ná.rio }
\end{aligned}
$$

No obstante, los llamados prefijos adjetivales sistemáticamente son elementos bisílabos terminados en vocal y llevan acento secundario en la primera sílaba. Es cierto que este comportamiento acentual no es una propiedad exclusiva de estos elementos frente a cualquier elemento clasificable como prefijo (cf. còn.tra.por.tá.da vs. *con. trà.por.tá.da), pero sí es sistemática entre los miembros de la clase. Nótese en (12d) que para que el comportamiento acentual no siga las reglas del acento rítmico no es necesario que la base sea reconocible como palabra independiente.

$$
\begin{aligned}
& \text { (12) a. mì.ni.re.lój }\left({ }^{*}\right. \text { mi.nì.re.lój) } \\
& \text { b. pà.ra.mi.lí.cia (*pa.rà.mi.lí.cia) } \\
& \text { c. prò.to.ban.tú }(* \text { pro.tò.ban.tú }) \\
& \text { d. hè.te.ro.gé.ne.o }\left({ }^{*}\right. \text { he.tè.ro.gé.ne.o) }
\end{aligned}
$$




\subsection{Propiedades semánticas}

Veamos ahora una segunda propiedad general a estos elementos. Como se ha adelantado ya, establecen relaciones semánticas asimilables a la modificación adjetival. Esto es inusitado dentro de la clase de los prefijos. (13), por ejemplo, no es parafraseable como 'una copa alta', pero (14) es parafraseable precisamente como 'un bus grande / pequeño'.

(13) sobre-copa

(14) $\{$ maxi-/mini-\}bús

No se conocen, hasta donde se nos alcanza, otros prefijos que establezcan esta clase de relación con su base; tampoco hay, de nuevo por lo que sabemos, sufijos de esta clase. Sin embargo, hay al menos una clase de compuestos productiva en español que establece con claridad esta relación entre sus miembros:

(15) a. pel-i-rroj-o ('(que tiene) pelo rojo')

b. brac-i-larg-o ('(que tiene) brazos largos')

c. cuell-i-cort-o ('(que tiene) cuello corto')

\section{3. ¿Por qué no son compuestos?}

Como prefijos, pues, los prefijos adjetivales son excepcionales en su fonología y en su semántica. Entonces, ¿por qué no se han clasificado como miembros dentro de compuestos? La razón es que hay razones al menos tan poderosas como las ahora expuestas para tratarlos como afijos. Repasemos los motivos.

Los miembros de un compuesto contienen al menos una raíz cada uno, y generalmente en español, uno o más afijos que marcan su adscripción categorial; ninguno de estos miembros está forzado a aparecer en el extremo de la derecha o en el de la izquierda del compuesto, como muestran las siguientes alternancias:

(16) a. poeta-pintor; pintor-poeta

b. verd-i-blanco; blanqu-i-verde

Frente a un miembro de un compuesto, un afijo tiene una posición fija, en el sentido de que, con respecto a la raíz, deberá situarse a su derecha o a su izquierda:

(17) a. contra-reloj; *reloj-contra

b. pre-fijo; *fijo-pre

Pues bien: ninguno de los elementos clasificados como prefijos adjetivales pueden ocupar otra posición que no sea la izquierda de la raíz, como se espera de un prefijo. No existen en español palabras complejas acabadas en paleo, mini, homo o pseudo, por ejemplo.

Otra propiedad formal de un miembro del compuesto es que o bien forma una estructura que puede funcionar como una unidad prosódica y formal en sintaxis (18), o bien en combinación con afijos puede hacerlo (19). 
$\begin{array}{ll}\text { (18) a. cuenta-cuentos } & \rightarrow \text { (Juan) cuenta cuentos } \\ \text { b. fil-ó-log-o } & \rightarrow \text { una fil-ia, la lóg-ica }\end{array}$

No es esto lo que sucede con ninguno de los elementos clasificados como 'prefijos adjetivales', lo cual de nuevo apoya el análisis de afijo, frente al de elementos compositivos:
(19) a. neo-: *neo-s, *neo-ura...
b. paleo-: *pale-íto, *super-paleo...
c. para-: *pár-ico, *pár-eza
d. pseudo-: *in-pseudo, *pséud-al
e. proto-: *prót-ico, *prot-idad...

Así pues, nos enfrentamos a un problema inicial: parece que tratemos como tratemos estos elementos, van a resultar excepcionales en algún sentido. Si los tratamos como prefijos, serían los únicos afijos, sufijo o prefijo, que establecen relaciones de modificación adjetival con sus bases; si los tratamos como miembros de un compuesto, serían los únicos con posición fija, e incapaces de formar estructuras independientes en combinación exclusiva con afijos. La situación es casi desesperanzadora. No obstante, en la siguiente sección vamos a argumentar que parte de la dificultad de encontrar una clasificación parsimoniosa de estos elementos radica en que no deben considerarse una sola clase homogénea; hay subcasos, y una vez que se identifican y se examinan sus propiedades, el problema de encontrarles un lugar natural en la teoría morfológica comienza a no parecer tan insalvable.

\section{Subclases de prefijos adjetivales}

En esta sección vamos a argumentar que, pese a compartir algunas propiedades que justifican su inclusión en una macro-clase, es necesario distinguir subgrupos que se definen por su comportamiento en su interior. Estos subgrupos resultarán cruciales para entender la naturaleza de estos elementos.

\subsection{Propiedades compartidas}

Comencemos con las propiedades que comparten estos prefijos, y que justifican que se pueda hablar de una clase definida. Las propiedades que se han discutido en $\$ 2$ y que los hacen problemáticos desde la perspectiva de la división entre derivación y composición también pueden considerarse compartidas por toda la clase.

\subsubsection{Orden}

Una primera característica común a todos los prefijos adjetivales es que son externos a lo que, si se quiere, podemos considerar prefijos prototípicos: tiempo y lugar. Si nos atenemos al conocimiento del mundo, podemos necesitar un término para hablar de un recinto que se encuentra detrás de una tienda, que es lo que expresa (20a). Tal vez queramos decir que ese recinto, aunque no parezca, no es en realidad tal, y así formamos la palabra de (20b); igualmente, podemos imaginar que queremos hablar 
de ese recinto trasero, pero ahora decir que se encuentra detrás de algo que, aunque parece una tienda, no lo es. Esta es la formación que tratamos de hacer en (20c), y como se ve, es marcadamente agramatical.
(20) a. tras-tienda
b. pseudo-tras-tienda
c. *tras-pseudo-tienda

Del mismo modo, podemos hablar de los preliminares por los que dos personas se comprometen a la firma de un contrato (21a). También podemos querer decir que de entre los documentos que preceden a ese contrato, estamos destacando el fundamental de ellos o, si se quiere, el primero de la serie, y esto es lo que expresa (21b). Podríamos querer referirnos, igualmente, a los preparativos que preceden al establecimiento de un protocontrato, pero esto es agramatical (21c).

(21) a. pre-contrato

b. proto-pre-contrato

c. *pre-proto-contrato

A continuación se muestran otros ejemplos similares.

(22) a. neo-post-colonial

b. *post-neo-colonial

(23) a. para-sub-marino

b. *sub-para-marino

(24) a. paleo-post-colonial

b. *post-paleo-colonial

Igualmente, los prefijos negativos y cuantificativos dan el mismo patrón de datos: nótese en (25b) que no hay nada incongruente en hablar de la negación de la propiedad de ser aparentemente útil, y sobre (26b), que igualmente nada impide decir que algo tiene tres válvulas de nueva generación, dado que podemos hablar de un artefacto de nueva generación que tiene tres válvulas.

(25) a. pseudo-in-útil

b. *in-pseudo-útil

(26) a. neo-tri-válvula

b. *tri-neo-válvula(s)

Veamos ahora los prefijos de actitud favorable o desfavorable:

(27) a. neo-anti-clerical

b. anti-neo-clerical

(28) a. contra-para-guerrilla ${ }^{2}$

b. para-contra-guerrilla

Cuando contra- tiene valor locativo, no se dan los mismos hechos de orden: compárese pseudo-contra-ventana $\mathrm{y}$ *contra-pseudo-ventana. 
(29) a. pseudo-pro-revolucionario

b. pro-pseudo-revolucionario

En este caso, ambas formaciones están permitidas. (28a), por ejemplo, se refiere a un grupo que reacciona contra una paraguerrilla, mientras que (28b) designa un grupo contraguerrillero alternativo. Es interesante recordar en este punto que los prefijos anti- y pro- se encuentran entre los que se consideran más externos a la estructura de la palabra, como muestra, por ejemplo, la propiedad a la que dan lugar de poder afectar a un sintagma completo: anti-[medidas de austeridad], pro-[movimientos migratorios].

De esta manera parece que tenemos una primera generalización (30a) y un dato sobre la interacción entre los prefijos de actitud (des)favorable y los adjetivales (30b):

(30) a. prefijos adjetivales / prefijos de actitud (des)favorable $>$ prefijos de negación, lugar, tiempo y cuantificación $>$ base

[Los prefijos adjetivales, al igual que los prefijos de actitud (des)favorable, nunca siguen a los prefijos de negación, lugar, tiempo y cuantificación]

b. Se admiten los órdenes 'prefijo de actitud (des)favorable > prefijo adjetival' y 'prefijo adjetival > prefijo de actitud (des)favorable'

Es interesante observar que los prefijos de de actitud (des)favorable también tienden a ser bisílabos (con excepciones: pro-), mientras que los de cantidad, tiempo, lugar y negación tienden a ser monosílabos (de nuevo, con excepciones: ante-, circum-). Esta es una propiedad interesante, que tal vez merezca una explicación, pero no puede ser tomada como base para una supuesta explicación prosódica del ordenamiento. Una secuencia como *ante-pro-aborto, que podría querer decir 'que precede en el tiempo a un movimiento a favor del aborto' es agramatical pese a que allí el elemento monosilábico es inmediatamente adyacente a la base.

El criterio que hemos desarrollado en esta sección permite comprobar que puede ser deseable incluir entre los prefijos adjetivales algunas formaciones que RAE \& ASALE (2009) incluyen en principio en otras clases, en virtud de su significado. Este es el caso de semi-, que se clasifica como cuantificativo y que por el mismo significado podría haber sido parafraseado como correspondiente al adjetivo 'medio, parcial':

(31) a. semi-pre-contrato

b. *pre-semi-contrato

(32) a. anti-semi-revolucionario

'contrario a quienes solo son parcialmente revolucionarios'

b. semi-anti-revolucionario

'parcialmente contrario a quienes defienden una revolución'

\subsubsection{Categoría gramatical de la base}

Una segunda observación es que todos estos prefijos admiten, regularmente, unirse a bases nominales, y rechazan su unión con bases verbales. Esta es, sin duda, una propiedad que comparten con los adjetivos, que, como es bien sabido, tienen propiedades formales que los asocian a los sustantivos y no a los verbos. 
De entre los adjetivos, lo que une a todos estos prefijos es que se unen con facilidad a adjetivos relacionales. Los adjetivos relacionales (véase Bosque 1993, 2006; Fábregas 2007) se han analizado como construcciones que contienen un sustantivo, y conservan la semántica básica del sustantivo en tanto que denotan una clase de objetos, y no una cualidad. En (33) se muestran algunos ejemplos con base sustantiva $\mathrm{y}$ con adjetivos relacionales.

(33) a. neo-barroco

b. paleo-cristiano

c. pseudo-renacentista

d. para-medicinal

e. proto-mártir

f. mini-reloj

g. maxi-aparcamiento

Con excepciones que serán examinadas en el apartado §3.2, los prefijos adjetivales no pueden combinarse con adjetivos calificativos; contrastan así neo-nazi (sustantivo o adjetivo relacional) con *neo-bondadoso; paleo-barroco con *paleoalambicado; semi-liberal con *semi-generoso.

(34) muestra que ninguno de estos prefijos admite combinación con bases verbales: se ofrece junto a la palabra imposible, como ayuda, una glosa de lo que razonablemente podría haberse esperado que significara la palabra, pero lo crucial es que la formación es agramatical en cualquier interpretación. (34j) muestra que semi- se comporta igual que los otros miembros de la clase, lo cual agrega mayor plausibilidad a la posibilidad de incluirlo aquí.

(34) a. *neo-escribir 'escribir de forma novedosa'

b. *paleo-estudiar 'estudiar de forma tradicional'

c. *pseudo-gobernar 'aparentar que se gobierna'

d. *para-investigar 'investigar de una forma alternativa'

e. *mini-construir 'construir cosas pequeñas'

f. *maxi-vestirse 'vestirse con cosas grandes'

g. *proto-inventar 'inventar por primera vez, inventar siendo el primero'

h. *homo-escribir 'escribir del mismo modo que otro, copiar'

i. *hetero-actuar 'actuar de forma heterodoxa, o diferente a los demás'

j. *semi-construir 'construir a medias'

\subsubsection{Generalizaciones semánticas}

Pese a que, como veremos, los prefijos adjetivales forman una clase heterogénea en virtud de muchas de sus propiedades, hay algunas nociones semánticas a las que suelen asociarse típicamente. Por una parte, destaca la proximidad de algunos de ellos a los adjetivos llamados 'adverbiales' en la bibliografía (por ejemplo, Demonte, 1999). Paleo- y neo- son similares -aunque, como veremos, no idénticos- al uso de adjetivos temporales como antiguo en el antiguo cristianismo y futuro en el futuro cristianismo, al menos en la medida en que incluyen en su denotación una noción clara de secuencia temporal. Pseudo- y para- se asemejan a los adjetivos intensionales que modifican la pertinencia de caracterizar un objeto con 
ciertas propiedades, como en una flor falsa o un aparente problema. Por su parte, proto- se asemeja o bien al adjetivo intensional perfecto, en tanto que expresa un miembro prototípico de una clase (un perfecto idiota), o al ordinal primero, que admite lecturas de prototipicidad (el primer artista). En par de oposición, cabe considerar semi- como adscripción solo parcial, no prototípica, a una clase, como en semi-liberal 'perteneciente a la clase de los liberales solo en parte'. Relacionados con la idea de identidad entre individuos o entre las clases a las que pertenecen los individuos tenemos homo- 'igual' (junto a los mucho menos productivos iso- y equi-) y hetero- 'diferente'.

Son algo diferentes los prefijos macro-, micro-, nano-, mini- y maxi-, que hablan de la propiedad física del tamaño, y los menos productivos cali- 'bueno, hermoso' y caco- 'malo, feo': estos son también adjetivos, pero nótese que en este caso el equivalente serían adjetivos calificativos, si bien adjetivos calificativos que podríamos considerar de campos semánticos básicos, como propiedades físicas fundamentales y valoraciones muy subespecificadas (bueno / malo).

Vemos aquí, de hecho, que ya hay motivos para sospechar que la clase de los prefijos adjetivales no es completamente homogénea; al menos, los adjetivos que expresan las propiedades correspondientes como formas autónomas se comportan de formas muy diferentes si expresan nociones temporales o de tamaño. En la siguiente sección veremos que de hecho existen diferencias independientes entre estos prefijos que permiten identificar, al menos inicialmente, ciertas subclases.

\subsection{Propiedades diferenciadoras}

Pasemos a las diferencias internas en el grupo.

\subsubsection{Propiedad de la base vs. propiedad de un pseudo-argumento de la base}

Comparemos lo que significa (35a) con lo que significa (35b).

(35) a. micro-economía

b. mini-economía

En el primer caso, el significado puede glosarse como 'la parte de la economía que se relaciona con fenómenos de escala menor'. Esta glosa no es aceptable para (35b), que debe entenderse propiamente como una economía pequeña. Es decir, en (35a) lo que es pequeño no es la economía, sino aquello que se relaciona con ella de algún modo (específicamente, en este caso, por nuestro conocimiento del mundo: lo que estudia la economía); en (35b), sí.

Este contraste no es aislado: considérese (36).

(36) a. homo-sexual

b. hetero-sexual

En biología, estas palabras pueden usarse para referirse literalmente a entidades del mismo o distinto sexo (como en dos especímenes homosexuales, 'dos especímenes del mismo sexo'). Sin embargo es más común darles la interpretación de 'relacionados (de algún modo) con el mismo sexo'. En ambas interpretaciones, la 
incidencia semántica del prefijo afecta a la base, ya sea al adjetivo sexual o al sustantivo sexo. No sucede lo mismo en (37):

(37) a. homo-plastia
b. heter-ó-trofo

Aquí el prefijo no modifica a la base, es decir, no estamos diciendo 'transplantes iguales' o 'alimentación distinta', sino 'transplante que se relaciona con individuos de la misma clase' o 'alimentación que se relaciona con individuos de distinta clase'; el prefijo adjetival califica propiedades no de la base, sino de una entidad que se presupone y que establece alguna relación con la noción semántica expresada por la base.

Los demás prefijos adjetivales admiten solo la primera lectura, en que modifican a la base, y rechazan la segunda, en que modifican a lo que parece ser un argumento implícito de la base:

(38) a. neo-plastia 'transplante novedoso', no *'transplante de algo nuevo'

b. pseudo-plastia 'transplante aparente', no *'transplante de algo aparente'

c. para-plastia 'transplante heterodoxo', no *'transplante de algo alternativo'

d. semi-plastia 'transplante parcial', no *'transplante de algo incompleto'

e. paleo-plastia 'transplante con método antiguo', no *‘...de algo antiguo'

Tenemos, pues, una primera división:

(39) a. Prefijos adjetivales que pueden aparecer en estructuras de lectura cuasiargumental: macro-, micro-, homo-, hetero-

b. Prefijos adjetivales forzados a una lectura de modificador: el resto

\subsubsection{Uso como adjetivo independiente}

Veamos ahora el contraste de (40); supongamos que acudimos a un establecimiento de comida rápida y pedimos una bebida de cierto tamaño, pero nos dan el tamaño contrario:

(40) a. Esta bebida no es mini.

b. Esta bebida no es maxi.

c. *Esta bebida no es macro.

d. *Esta bebida no es micro.

e. *Esta bebida no es nano.

Lo que comprobamos aquí es que algunos de los llamados prefijos adjetivales en realidad admiten, si bien de manera restringida, un uso como formas libres. Este uso se admite tanto en posición predicativa (40) como atributiva (41), si bien en ambos casos está restringido a artefactos y otros seres no animados:

(41) a. Tengo un ordenador mini.

b. Tengo un coche maxi.

c. *Tengo una mascota mini.

Asimismo, frente a los adjetivos grande y pequeño, parece que para poder utilizar mini y maxi como formas libres, debe presuponerse que existe una serie de tamaños 
estandarizados de un artefacto tal que cada tamaño defina una categoría dentro del objeto. Si suponemos que una empresa comercializa un modelo de ordenador en varios tamaños (42), el menor de ellos puede describirse como mini, y el mayor como maxi, pero cuando nuestro conocimiento del mundo nos dice que no es normal que se diseñen los objetos modificados conforme a estos parámetros (como en 43), la secuencia es marcada. (43a) se salva si damos por hecho que alguna empresa ha comercializado, a través de procedimientos de ingeniería genética, sandías en dos tamaños estandarizados que se salen ambos del tamaño habitual de las sandías; en tal caso, estamos reinterpretando la sandía, que es un objeto natural, como un artefacto fruto del diseño consciente.

(42) a. un ordenador mini

b. un ordenador maxi

(43) a. \#una sandía mini

b. \#un pepino maxi

c. un bocadillo mini

Esto es una propiedad exclusiva de un subgrupo dentro de los prefijos adjetivales que designan nociones asimilables a los adjetivos calificativos. El resto de prefijos rechazan estos usos como formas libres, incluso en posición atributiva. En (44) incluimos junto a la forma agramatical una paráfrasis razonable de lo que podría significar, que sí es gramatical, para mostrar que el problema no es semántico.

(44) a. *un problema pseudo (cf. 'un problema aparente')

b. *un hombre proto (cf. 'un hombre prototípico')

c. *un nazi neo (cf. 'un nazi moderno, un nazi nuevo')

d. *un liberal semi (cf. 'un liberal parcial, un liberal moderado')

\subsubsection{Modificación de adjetivos calificativos y nombres propios}

Hemos visto que todos los prefijos adjetivales admiten bases nominales (nombres comunes y a menudo también adjetivos relacionales de base nominal) y rechazan bases verbales. No obstante, uno de los prefijos presenta un comportamiento divergente del resto en la medida en que admite también adjetivos calificativos y nombres propios. Obsérvese (45), donde se muestran ejemplos reales de textos de internet:

(45) a. La recepcionista interrumpe el devore de sus pingüinos, los deja a un lado; luego, con voz aguda pseudo amable inquiere...

b. semana pseudo-feliz

c. tuve que organizar alguna especie de ritual pseudo divertido chistoso que le resulte placentero

En estos casos, el prefijo adjetival modifica a un adjetivo calificativo en el que no cabe suponer una base nominal. Si comprobamos ahora la posibilidad de combinarse con nombres propios, comprobamos que este prefijo también lo permite:

(46) el pseudo-Dante ('alguien posiblemente identificado con Dante') 
Observando el ejemplo en detalle, se pueden observar tres aspectos destacables. El primero es que, efectivamente, en estos casos parece que el nombre ya no tiene el comportamiento gramatical esperable de un nombre propio: el artículo definido es necesario. Los casos en que pseudo- aparece en combinación con el nombre propio, pero sin artículo, son casos en que el prefijo ya forma parte del nombre propio, como en Pseudo-Dionisio Areopagita. No obstante, y este es el segundo factor, no ha habido una recategorización semántica del sustantivo, en el sentido de que el nombre sigue refiriéndose a una entidad, y no a un conjunto de propiedades (frente a lo que sucede, por ejemplo, en 47). Por este motivo tenemos la tercera propiedad, que es que en estos casos pseudo- tiene que referirse a la identidad, y no a las propiedades del sustantivo, que sobre suposiciones estándar acerca del significado de un nombre propio (Kripke 1971), están ausentes.

La modificación de nombres propios es imposible para los demás prefijos adjetivales, incluso cuando la noción que expresan puede ser compatible con la noción de identidad:

(47) a. *el homo-Pedro (cf. 'el mismo Pedro [que antes]')

b. *un hetero-Pedro (cf. 'un Pedro distinto [al de antes]')

c. *el neo-Shakespeare (cf. 'el nuevo Shakespeare, una persona nueva llamada igual')

$(47 \mathrm{c})$ es aceptable marginalmente si Shakespeare se recategoriza como un predicado que contiene el conjunto de rasgos semánticos que se considera que Shakespeare manifiesta por antonomasia, en estructuras del tipo de Está hecho todo un Shakespeare. ${ }^{3}$

Parece haber, intuitivamente, una motivación semántica para la distribución categorial más flexible de pseudo-: la noción que expresa este prefijo se acerca a la modalidad evidencial ('pese a la apariencia, el elemento no pertenece a esa clase'), y en su paráfrasis puede encontrarse un equivalente en la forma aparentemente, que es un adverbio y por tanto puede modificar a adjetivos. De manera interesante, la noción de apariencia puede aplicarse tanto a relaciones de identidad como a propiedades, y en algunos casos, de hecho puede expresarse con el adverbio:

(48) a. Aparentemente, es el Papa. (nombre propio)

b. el aparentemente culpable (nombre común)

c. aparentemente sucio (adjetivo calificativo)

Esta equivalencia entre una paráfrasis adjetival y una paráfrasis adverbial no se extiende a otros prefijos adjetivales: por ejemplo, grandemente no puede referirse al tamaño físico de un objeto.

3 Un revisor anónimo, al que agradecemos la observación, nota que otros prefijos pueden combinarse con sustantivos como renacimiento y romanticismo. Aunque es cierto que estos sustantivos comparten con los nombres propios parte de su comportamiento semántico - denominan e identifican-, nótese que su comportamiento sintáctico es muy distinto. Así, mientras Dante -como los nombres propios más prototípicos- puede ser sujeto preverbal sin determinante, esto no es posible con estos sustantivos.

(i) a. Dante murió entonces.

b. *(El) renacimiento murió entonces. 


\subsubsection{Orden relativo entre los prefijos}

Por último, si bien con respecto a otras clases de prefijos todos los prefijos adjetivales se comportan igual, la concurrencia de más de un prefijo adjetival está regida por algunos hechos de orden.

Consideremos primero el orden relativo entre neo- y los que designan relaciones argumentales. Podemos concebir una nueva forma de economía a la que nos referiríamos con (49a), y también podemos hablar de la economía que estudia los fenómenos a pequeña escala (49b); podemos hablar de una nueva versión de la microeconomía con la forma de (49c), pero aunque podamos concebir una nueva economía que trate los fenómenos a pequeña escala, (49d) es agramatical.
(49) a. neo-economía
b. micro-economía
c. neo-micro-economía
d. *micro-neo-economía

Igualmente sucede con el prefijo pseudo- y otro de los que hemos clasificado como capaces de expresar relaciones argumentales: podemos hablar de algo que solo en apariencia es homorgánico (50a), pero (50b) es imposible pese que podemos concebir una entidad aparentemente orgánica que sea igual a otra o establezca una relación con otra del mismo tipo.

(50) a. pseudo-homo-orgánico

b. *homo-pseudo-orgánico

Entre mini-, ejemplo de prefijo que puede usarse como forma libre, y neo- se encuentran también restricciones de orden: podemos hablar de un nazi de nueva generación (51a), o de un nazi de tamaño diminuto (51b); también podemos hablar de un nazi de nueva generación que es diminuto (51c), pero (51d) es imposible aunque podamos hablar de nazis pequeños de nueva generación.
(51) a. neo-nazi
b. mini-nazi
c. mini-neo-nazi
d. *neo-mini-nazi

Aunque tanto mini- como pseudo- puedan preceder a neo-, no están en orden libre. Aunque cabe hablar de un objeto pequeño que aparente ser un reloj pero no lo termine de ser, (52a) es imposible, frente a (52b). Nótese que en (52b), como esperamos, cabe la posibilidad de que el objeto sí sea un reloj, pero lo que el hablante sugiera que es una denominación apropiada es que sea un reloj lo bastante pequeño.
(52) a. *mini-pseudo-rreloj
b. pseudo-mini-rreloj

Por tanto, pseudo- precede a mini-; (53) muestra que hay transitividad en la relación de precedencia, ya que pseudo- también ha de preceder a neo-: 
(53) a. pseudo-neo-barroco

b. *neo-pseudo-barroco

Obtenemos así una secuencia como la de (54):

(54) pseudo- > mini- > neo- > homo-, micro-

\section{Análisis: generalizaciones y propiedades}

Las propiedades que acabamos de revisar no se distribuyen de forma caótica, y permiten definir cuatro clases -al menos- de prefijos adjetivales, donde se observan ya ciertas líneas de análisis, algunas de las cuales exploraremos.

(55) Clases:

a. Prefijos adjetivales capaces de expresar relaciones cuasi-argumentales: homo-, hetero-, macro-, micro-. Deben ir más unidos a la base que el resto de las subclases de prefijos adjetivales; solo se combinan con nombres comunes $\mathrm{y}$ adjetivos relacionales. No pueden usarse como formas libres.

b. Prefijos adjetivales neutrales o no marcados: neo-, paleo-... No expresan relaciones argumentales, están restringidos a la combinatoria con nombres comunes y adjetivos relacionales; no pueden usarse como formas libres, y preceden a los de la clase anterior.

c. Prefijos adjetivales libres: mini-, maxi-. No expresan relaciones argumentales, se combinan solo con nombres comunes y adjetivos relacionales, y preceden a los de las dos clases anteriores.

d. Pseudo-: no expresa relaciones argumentales, son los más externos de la serie y pueden combinarse con adjetivos calificativos y nombres propios.

Hay varios hechos que son interesantes aquí. El primero es que las propiedades de cada clase se organizan casi perfectamente como una escala de implicación unidireccional, como ilustramos en la siguiente tabla:

Tabla 1. Implicaciones entre las propiedades

\begin{tabular}{|l|c|c|c|c|}
\cline { 2 - 5 } \multicolumn{1}{c|}{} & Micro- & Neo- & Mini- & Pseudo- \\
\hline Relaciones argumentales & Sí & No & No & No \\
\hline Forzado a aparecer con una base & Sí & Sí & No & Sí \\
\hline Restringido a N común y A relacional & Sí & Sí & Sí & No \\
\hline
\end{tabular}

Las propiedades del prefijo micro- son, pues, un subconjunto de las de neo-, y las de neo- lo son con respecto a las de mini-. Hemos destacado una casilla, que es la que en principio impide concebir esta tabla como una secuencia de propiedades que se implican en una dirección: el prefijo pseudo- no puede aparecer como forma libre, por lo cual no cabe decir que sus propiedades sean un superconjunto de las de mini-. 
No obstante, es plausible pensar que hay motivos independientes para que pseudono aparezca como forma libre: sabemos que la semántica que tiene es intensional, y muchos adjetivos intensionales rechazan la posición post-nominal y la construcción predicativa (56):

(56) a. un presunto culpable

b. ?? un culpable presunto

c. *El culpable es presunto.

Tal vez quepa proponer que realmente si atendemos a su carácter estructural y morfosintáctico, pseudo- podría funcionar como una forma libre, y es solo la semántica asociada a él lo que le impide aparecer en contextos donde esta propiedad sea visible directamente.

En cualquier caso, incluso con esta pregunta abierta, es fácil comprobar que cuanto más próximo a la base sea un prefijo dentro de las subclases de prefijos adjetivales, más acceso tiene a condiciones típicamente léxicas de la base: en el caso extremo, puede manipular o acceder de algún modo a su estructura argumental, para modificar a las entidades que podrían considerarse argumentos de esa base. En tal caso, está forzado a no ser nunca forma libre, y a estar muy restringido categorialmente. En el lado opuesto, pseudo- tiene una libertad casi total en la clase de bases a las que puede unirse, pero no puede expresar relaciones cuasi-argumentales. Lo que esto sugiere es que las distintas clases están distribuidas estructuralmente, con aquellas más próximas a la base introducidas por proyecciones del sustantivo que actúa como núcleo en las que aún se puede acceder a su significado léxico, mientras que las más externas estarían legitimadas por estructuras funcionales que no remiten específicamente a ninguna clase léxica determinada.

A continuación, como ilustración de esta teoría general, y sin ánimo exhaustivo, vamos a discutir con algo más de detalle los prefijos adjetivales micro-, neo- y pseu$d o-$, para mostrar cómo las intuiciones que han surgido de la descripción de los datos son formalizables en una teoría neoconstruccionista.

Un ingrediente central dentro de nuestra propuesta es la propuesta de que los prefijos adjetivales son especificadores de distintas proyecciones funcionales dentro del área del sustantivo, de tal manera que cuanto más externos sean a la base léxica más flexibles son en su comportamiento. Como se verá, propondremos que los dos aspectos extraordinarios de los prefijos adjetivales desde la perspectiva de la prefijación en general se siguen de la misma propiedad: estos prefijos son especificadores de categorías que jerárquicamente dominan a $\mathrm{SN}$, la proyección donde se define el conjunto de propiedades denotado por un sustantivo léxico. Siendo especificadores, y no núcleos, sus propiedades prosódicas quedan explicadas automáticamente partiendo de una teoría en la que el especificador debe constituir un dominio cerrado desde la perspectiva de la sintaxis y la fonología (Uriagereka, 1999): entre otras propiedades bien conocidas, los especificadores no permiten extracciones sintácticas. La propuesta de que son especificadores de proyecciones distintas a $\mathrm{SN}$, frente a núcleos asimilables a las preposiciones, como sería el caso de los prefijos de tiempo y lugar (Fábregas, 2010), explica también la segunda propiedad anómala: estos prefijos son modificadores de distintas nociones, relacionadas con la denotación de la proyección en la que se incluyen, por lo que toman al complemento de dicha proyección como el predicado modificado. Por último, al ser especificadores de categorías más altas que 
SN, se predice automáticamente que preceden a los prefijos prototípicos, que forman estructuras con $\mathrm{SN}$.

En (57) hacemos explícitas nuestras suposiciones sobre la jerarquía interna entre las proyecciones relevantes.

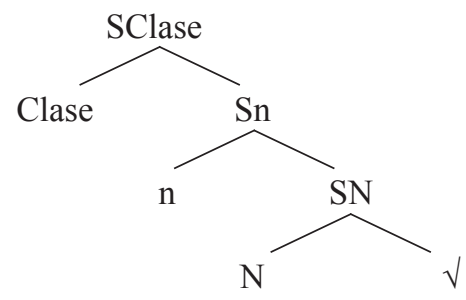

Junto a SN, que categoriza una raíz desprovista de información gramatical (cf. Marantz, 1997; Borer, 2013), tenemos Sn. Sn es la proyección que se encarga de convertir el conjunto de propiedades denotadas por SN en una entidad individual con un índice de identidad que pueda permitir hacer juicios sobre su identidad o diferencia con respecto a otras entidades (Baker, 2003). Por encima de Sn encontramos SClase (Zamparelli, 2000), que se responsabiliza de convertir esa entidad individual en una clase de elementos que puede tener uno o más miembros individuales en su interior.

Nótese que esta misma jerarquía es la que se hace necesaria para generar una secuencia como esta especie de animal, donde animal es un $\mathrm{Sn}+\mathrm{SN}$, es decir, un conjunto de propiedades con índice de identidad, mientras que especie es una manifestación analítica de SClase.

\subsection{Micro-: su naturaleza estructural}

Comencemos con la propuesta de análisis para micro-, que usaremos como ilustración de la clase de elementos que pueden modificar no la noción expresada por la base, sino un elemento que establece una relación con ella.

Véase la estructura de (58).

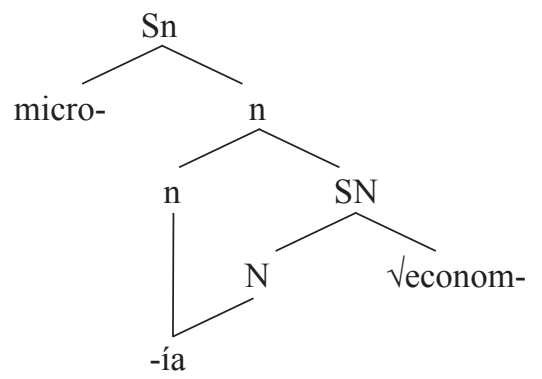

Como se ve, en esta estructura proponemos que una raíz se convierte en sustantivo por la adición de SN, que, junto a Sn se materializa como un sufijo nominalizador que diferencia econom-ía de, por ejemplo, económ-ico o econom-ista. Veamos ahora los motivos por los que micro- se introduce en el especificador de Sn y no otra proyección.

La proyección Sn se ha asociado en numerosos trabajos (Lecarme 2008, por ejemplo), con la proyección que es responsable de introducir un argumento relacionado con el sustantivo base, en su complemento SN. En este sentido, se ha propuesto que $\mathrm{Sn}$ es el equivalente nominal a Sv e introduce como argumento ex- 
terno, por ejemplo, el complemento semánticamente obligatorio de los sustantivos relacionales que expresan parentesco, como madre. Al introducir el prefijo como especificador de esta categoría, pues, garantizamos que su interpretación pueda afectar no a la base nominal en SN, sino al argumento implícito que se relaciona con dicha noción, que es exactamente lo que queremos de la denotación de micro-.

El hecho de que esta clase de prefijos solo pueda unirse a sustantivos y adjetivos relacionales, con base nominal, se sigue necesariamente si son modificadores de Sn: ni los adjetivos calificativos ni los verbos contienen esta proyección funcional, porque es exclusiva del área del sustantivo.

Recuérdese también que siguiendo a Baker (2003), Sn es la proyección que introduce el índice de identidad asociado al individuo denotado por un sustantivo. Esto explica que los prefijos homo- y hetero-, que precisamente requieren un juicio sobre la identidad o diferencia entre dos entidades con ciertas propiedades, puedan expresar propiedades de las nociones relacionadas con la base: en virtud de la posición en la que se introducen debido a su significado, también se sigue inmediatamente que deben ser capaces de esta operación argumental, precisamente porque $\mathrm{Sn}$ es también la proyección que define las relaciones de la base con sus argumentos nominales.

\subsection{Neo-: su naturaleza estructural}

Pasando ahora al segundo prefijo, neo-, obsérvese en (59) que estamos proponiendo que este es introducido como especificador de SClase, la proyección responsable de convertir al individuo designado por Sn en un conjunto de individuos definido por ciertas propiedades.

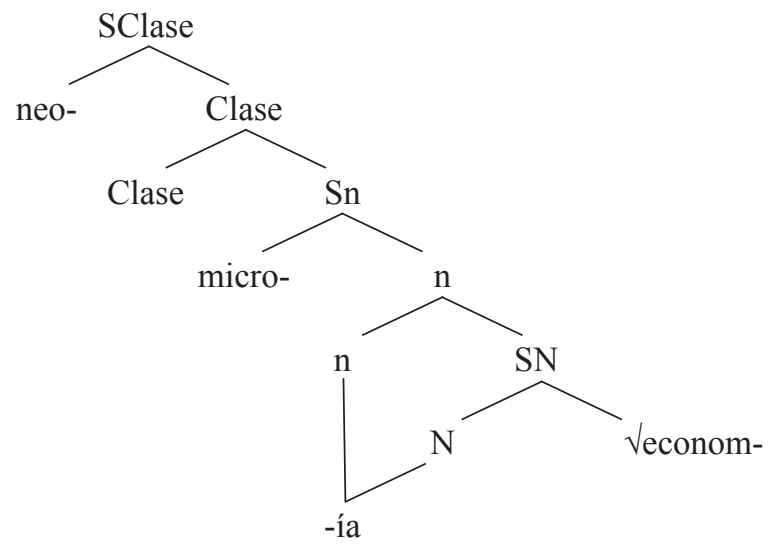

Esta hipótesis automáticamente explica el orden entre los dos prefijos en una palabra como neo-micro-economía.

Para mostrar por qué es plausible que neo- ocupe precisamente esta noción, comparemos la denotación de (60a) con (60b).

(60) a. un nuevo presidente

b. un neo-presidente

(60a) produce entrañamientos que no están incluidos en (60b). Para interpretar correctamente (60a) es necesario suponer que tenemos una secuencia de presidentes, en primer lugar. Esto no es necesario en (60b), donde podríamos estar refiriéndonos a la primera persona que actúa como presidente de una institución que se acaba de fundar. 
En segundo lugar, la secuencialidad temporal que implica (60a) requiere que estemos dando por hecho la existencia de distintos referentes que han desempeñado la misma función. Por el contrario, en (60b) podríamos estar hablando de un mismo referente, que acaba de ser presidente y ahora, por emplear un estilo distinto de presidencia que permite clasificarlo como un presidente de nueva generación política, ha pasado a ser un neo-presidente. En otras palabras, (60b) no nos dice nada sobre la secuencia de entidades o los referentes que han desempeñado el papel de presidente: nos dice, en cambio, qué clase de presidente es la relevante en este contexto, para restringir su denotación a solo el conjunto de propiedades que caractericen un estilo distinto, 'nuevo', de ejercer la presidencia. Esta interpretación es precisamente la que esperamos si el prefijo es modificador de SClase: que defina una subclase particular, en este caso, por la semántica del prefijo, una que sea nueva con respecto a un estilo anterior presupuesto.

Al igual que en el caso anterior, esperamos que estos prefijos solo se combinen con bases nominales o adjetivos relacionales, dado que solo estos pueden contener SClase.

\subsection{Pseudo-: su naturaleza estructural}

Pasemos, por último, a pseudo-, que proponemos es introducido como un especificador de una categoría funcional que se relaciona con la noción de modalidad y que es externa a todas aquellas que definen la proyección extendida de una categoría léxica. En esta propuesta, estamos siguiendo intuiciones que se han desarrollado en DeLazero (2011) y Sakyi (2013), que han estudiado los adjetivos modales. La propuesta en ambos autores es que en el interior de un sintagma nominal, un adjetivo modal tiene que terminar dando las mismas condiciones de verdad que un adverbio adjunto a la cláusula. Para esto es necesario que haya una interpretación proposicional encubierta del sintagma nominal (DeLazero, 2011), algo que en su teoría semántica se obtiene con una operación de cambio de tipo que reinterpreta el conjunto de propiedades denotadas por el sustantivo como una adscripción de propiedades (o de denominación) a un referente, de tal manera que el sintagma nominal se reinterpreta como una situación. Nuestra propuesta es sintáctica, pero construye sobre la de estos autores. El elemento modal aparece introducido en una posición periférica del SN, de manera que el operador semántico (esquema, siguiendo a DeLazero, 2011) que convierta al sustantivo en una situación quede jerárquicamente por debajo de él.

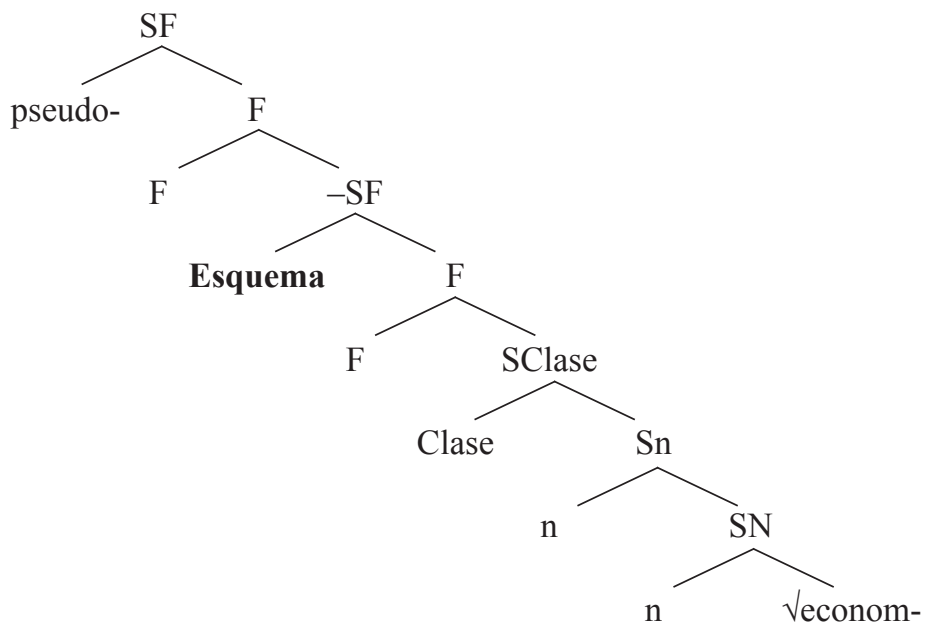


(62) muestra que esta hipótesis da cuenta automáticamente del orden entre prefijos.

(62)

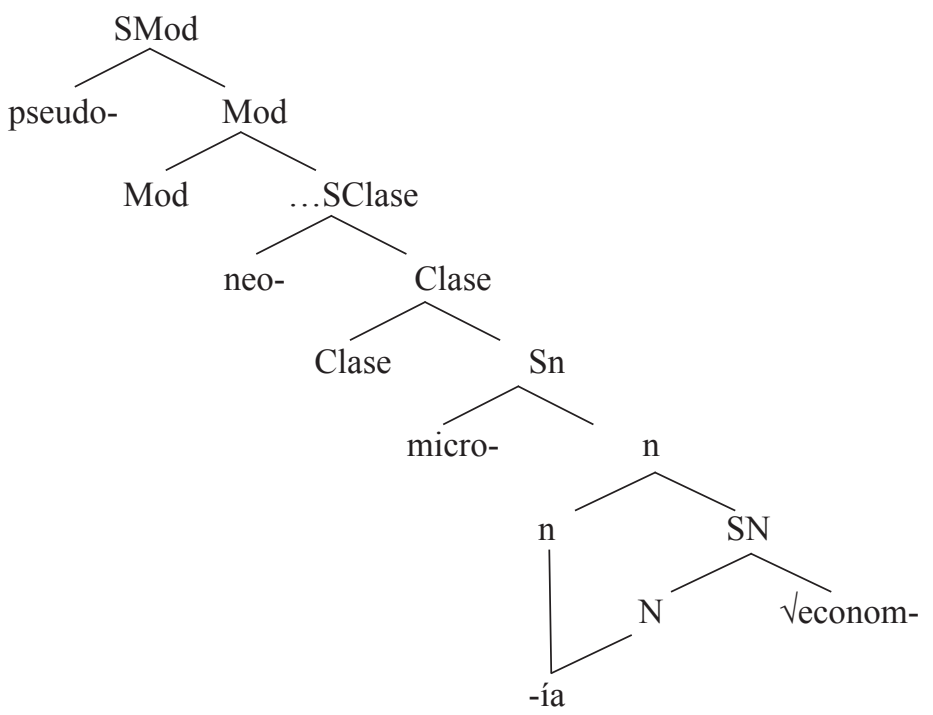

La noción de modalidad, como se ha mostrado antes, es independiente de la categoría léxica de la base; esto se captura en la estructura permitiendo que la modalidad sea una categoría subespecificada categorialmente que, por esta razón, es externa a las categorías funcionales nominales. Por esta razón, pseudo- tiene una combinatoria más libre que el resto de prefijos, ya que no es introducido por un núcleo exclusivamente nominal.

\section{Conclusiones: consecuencias para la división entre composición y prefijación}

Recapitulando lo que hemos argumentado aquí, parece que la división de los prefijos adjetivales en subclases permite clarificar su posición dentro del contraste entre composición y derivación. El examen más detallado de sus propiedades nos ha permitido distinguir cuatro clases; de ellas, una (mini- y maxi-) permiten el uso del exponente como adjetivo libre, lo cual sugiere que deberían considerarse más bien como formantes compositivos que como prefijos. Las otras clases que hemos identificado se comportan como esperamos de un especificador de una proyección funcional por encima de la base sustantiva; esto permite explicar su independencia fonológica y su semántica, bajo la premisa estándar de que los especificadores son dominios cerrados y se emplean precisamente para modificar las nociones expresadas en sus núcleos. Más allá de esto, la hipótesis desarrollada en las últimas secciones permite relacionar también su capacidad de modificar a pseudoargumentos de la base o no con su orden linear y su sensibilidad a la categoría léxica de la base.

Este trabajo tiene una naturaleza programática en el sentido de que su objetivo es el de establecer criterios formales que permitan identificar un prefijo adjetival, dada la vaguedad de una definición que solo se apoye en la equivalencia con un 
adjetivo. Secundariamente, el trabajo ha permitido identificar subclases de estos prefijos, pero no se ha proporcionado un análisis completo de cada una de estas clases, dado que la atención se ha concentrado en cómo diferenciarlas. No obstante, esperamos haber podido contribuir al campo con estos nuevos criterios y generalizaciones para permitir que esta clase de prefijos puedan ser abordados desde una perspectiva más formal.

\section{Obras citadas}

Almela, Ramón, Procedimientos de formación de palabras en español, Barcelona, Ariel, 1999

Baker, Mark, Lexical categories, Cambridge, Cambridge University Press, 2003.

Borer, Hagit, Taking form. Volumen 3 de The Exoskeletal Trilogy, Oxford, Oxford University Press, 2013.

Bosque, Ignacio, "Sobre las diferencias entre los adjetivos relacionales y los calificativos", Revista Argentina de Lingüística, vol. 9 (1993), pp. 9-48.

Bosque, Ignacio, "Coordinated Adjectives and the Interpretation of Number Features", en Laura Brugè (ed.), Studies in Spanish Syntax, Venecia, Università Ca' Foscari Venezia, 2006, pp. 47-60.

Demonte, Violeta, "El adjetivo: clases y usos", en Ignacio Bosque y Violeta Demonte (dirs.), Gramática descriptiva de la lengua española, Madrid, Espasa, 1999, pp. 129-217.

Delazero, Octav Eugen, "On the semantics of modal adjectives", University of Pennsylvania Working Papers in Linguistics, vol. 17 (2011), pp. 87-94.

Di Sciullo, Anna Maria, Asymmetry in Morphology, Cambrdige (Mass.), MIT Press, 2005

Fábregas, Antonio, "The internal syntactic structure of relational adjectives", Probus, 19 (2007), pp. 1-36.

Fábregas, Antonio, “A cartographic analysis of Spanish prepositional prefixes", Catalan Journal of Linguistics, 9 (2010), pp. 55-77.

Fábregas, Antonio \& Sergio Scalise, "The head in compounding”, en Sergio Scalise \& Irene Vogel (eds.), Cross-disciplinary issues in Compounding, Amsterdam, John Benjamins, 2010, pp. 109-127.

Kripke, Saul, Naming and necessity, Boston, Basil Blackwell, [1971] 1980.

Lecarme, Jacqueline, "Tense and modality in nominals", en Jacqueline Guéron \& Jacqueline Lecarme (eds.), Time and modality, Dordrecht, Springer, 2008, pp. 195-225.

Lieber, Rochelle \& Pavol Stekauer (eds.), The Oxford Handbook of Compounding, Oxford, Oxford University Press, 2009.

Marantz, Alec, "No escape from syntax: don't try morphological analysis in the privacy of your own lexicon", University of Pennsylvania Working Papers in Linguistics, 4 (1997), pp. 188-195.

Montero Curiel, María Luisa, La prefijación negativa en español, Cáceres, Servicio de Publicaciones, 1999.

RAE \& ASALE, Nueva gramática de la lengua española, Madrid, Espasa, 2009.

Sakyi, Joana Portia, "Modal adverbs and predicative modal adjectives in Akan", Selected Proceedings of the Annual Conference on African Linguistics, 43 (2013), pp. 9-48.

Scalise, Sergio, Antonietta Bisetto \& Emiliano Guevara, "Selection in compounding and derivation", en Wolfgang Dressler et al. (eds.), Morphology and its demarcations, Amsterdam, John Benjamins, 2005, pp. 133-150. 
Stehlík, Petr, Aspectos problemáticos de la prefijación en español, Brno, Masarykova Univerzita, 2011.

Uriagereka, Juan, “Multiple spell out”, en Norbert Hornstein \& Samuel Epstein (eds.), Working Minimalism, Cambridge (Mass.), MIT Press, 1999, pp. 251-282.

Varela, Soledad \& Josefa Martín García, "La prefijación”, en Ignacio Bosque \& Violeta Demonte (dirs.), Gramática descriptiva de la lengua española, Madrid, Espasa, 1999, pp. 4993-5041.

Zamparelli, Roberto, Layers in the determiner phrase, Nueva York, Garland, 2000. 